\title{
Relation between Oscillometric Measurement of Central Hemodynamics and Left Ventricular Hypertrophy in Hypertensive Patients
}

\author{
Masakazu Obayashi ${ }^{a}$ Shigeki Kobayashi ${ }^{b}$ Takuma Nanno $^{a}$ Yoriomi Hamada $^{a}$ \\ Masafumi Yano ${ }^{\text {b }}$ \\ aDepartment of Cardiovascular Medicine, Sanyo-Onoda City Hospital, Sanyo-Onoda, Japan; ${ }^{b}$ Division of Cardiology, \\ Department of Medicine and Clinical Science, Yamaguchi University Graduate School of Medicine, Ube, Japan
}

\section{Keywords}

Central systolic blood pressure · Augmentation index · Left ventricular hypertrophy

\begin{abstract}
Introduction: The augmentation index (Alx) or central systolic blood pressure (SBP), measured by radial applanation tonometry, has been reported to be independently associated with left ventricular hypertrophy (LVH) in Japanese hypertensive patients. Cuff-based oscillometric measurement of the Alx using Mobil-O-Graph ${ }^{\circledR}$ showed a low or moderate agreement with the Alx measurement with other devices. Methods: The Alx measured using the Mobil-O-Graph was validated against the tonometric measurements of the radial Alx measured using HEM-9000Al in 110 normotensive healthy individuals (age, 21-76 years; 50 men). We investigated the relationship between the central hemodynamics assessed using the Mobil-O-Graph and LVH in 100 hypertensive patients (age, 54-75 years; 48 men), presenting a wall thickness of $\geq 11 \mathrm{~mm}$ and $\geq 10 \mathrm{~mm}$ in men and women, respectively. Results: Although the Mobil-O-Graph-measured central Alx showed no negative values, it correlated moder-
\end{abstract}

ately with the HEM-9000Al-measured radial Alx $(r=0.602$, $p<0.001$ ) in the normotensive individuals. The hypertensive patients did not show a significant difference in the central SBP between the sexes, but the central Alx was lower in men than in women. The independent determinants influencing left ventricle (LV) mass index (LVMI) $\left(R^{2}=0.362\right.$; adjusted $R^{2}$ $=0.329, p<0.001)$ were heart rate $(\beta=-0.568 \pm 0.149, p<$ $0.001)$, central SBP $(\beta=0.290 \pm 0.100, p=0.005)$, and aortic root diameter $(\beta=1.355 \pm 0.344, p=0.001)$. Age $(\beta=-0.025$ $\pm 0.124, p=0.841)$ and the central Alx $(\beta=0.120 \pm 0.131, p=$ 0.361 ) were not independently associated with the LVMI. The area under the receiver operator characteristic curve to evaluate the diagnostic performance of the central Alx for the presence of LVH (LVMI $>118 \mathrm{~g} / \mathrm{m}^{2}$ in men or $>108 \mathrm{~g} / \mathrm{m}^{2}$ in women) was statistically significant in men $(0.875, p<$ 0.001 ) but not in women $(0.622, p=0.132)$. In men, a central Alx of $28.06 \%$ had a sensitivity of $83.3 \%$ and specificity of $80.0 \%$ for detecting LVH. Conclusions: Alx measurement in men provided useful prognostic information for the presence of LVH. Pulse-wave analysis assessed using the MobilO-Graph may be a valuable tool for detecting LVH in hypertensive patients.

(c) 2021 S. Karger AG, Basel karger@karger.com www.karger.com/pls
(C) 2021 S. Karger AG, Basel

Karger"
Correspondence to:

Masakazu Obayashi, mobayash@gmail.com 


\section{Introduction}

With age, the central blood pressure (BP) increases in the systole and decreases in the diastole, owing to the augmentation of late systolic pressure. The development of left ventricular hypertrophy (LVH) is attributed to the hemodynamic overload of the left ventricle (LV). It has been shown that stiffening of the proximal aorta [1] and high wave reflection $[2,3]$ lead to LVH. In particular, increased wave reflection induces LVH by increasing the late systolic loading $[4,5]$.

The augmentation index (AIx) is the ratio of the augmentation pressure (AP) to pulse pressure. Reportedly, the degree of LVH was independently correlated with the AIx of the radial artery measured by applanation tonometry in untreated patients with hypertension [6]. Recently, an elevated AIx was attributed to not only higher wave reflection but also increased forward wave propagation due to increased LV ejection [7] or decreased aortic reservoir function [8]. Therefore, the inefficacy of the AIx as an index of wave reflection in LVH was reported [9]. Moreover, a previous study performed using radial applanation tonometry reported that central systolic BP (SBP) was an independent parameter of LVH in Japanese hypertensive patients [10].

The Mobil-O-Graph ${ }^{\circledR}$ Pulse Wave Analysis Monitor (IEM GmbH, Stolberg, Germany) is an oscillometric device for measuring central BP using the ARCSolver method, which reconstructs the central pressure waveform by applying a generalized transfer-function method [11]. With respect to the AIx measurement, the Mobil-OGraph showed a low agreement with other devices measuring the central BP, such as those using the oscillometric (Arteriograph) and piezoelectric (Complior) techniques [12]. In contrast, the measurement of the AIx with an Arteriograph was reportedly related closely to that of the tonometric technique (SphygmoCor) [13]. There was a moderate agreement between the SphygmoCor single measurement and the Mobil-O-Graph 24-h mean value of the AIx [14].

Our previous study showed that the brachial cuffbased waveform recordings using the Mobil-O-Graph are feasible for the estimation of central AP in normotensive Japanese individuals [15]. Few studies have evaluated the correlation between the central SBP or AIx measured using the Mobil-O-Graph and LVH [16]. This study assessed the relationship between the central hemodynamics assessed using the Mobil-O-Graph and LVH in hypertensive patients.

Oscillometric Measurement of the

Augmentation Index

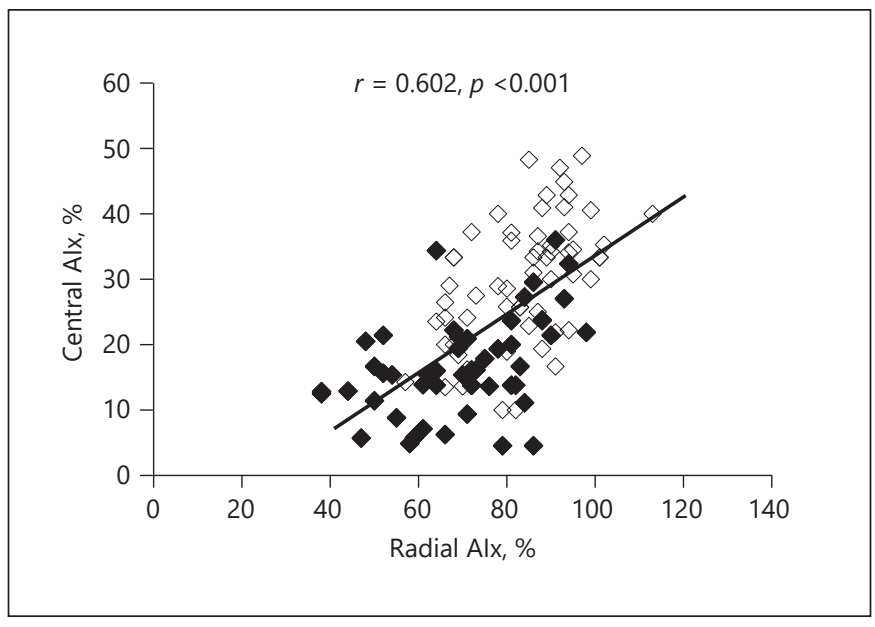

Fig. 1. Pearson correlation between the central AIx measured by Mobil-O-Graph and the radial AIx measured by HEM-9000AI in 110 normotensive healthy individuals. The regression line is shown as a solid line; black diamond shapes represent men and open diamond shapes represent women. AIx, augmentation index.

\section{Materials and Methods}

\section{Patients}

This study included never-treated hypertension patients and those undergoing antihypertensive treatment. Hypertension was diagnosed at outpatient clinics or during medical checkups as SBP $\geq 140 \mathrm{~mm} \mathrm{Hg}$ or diastolic BP (DBP) $\geq 90 \mathrm{~mm} \mathrm{Hg}$. The exclusion criterion was a history of coronary artery disease, heart failure, arrhythmia, or diabetes.

\section{Indices of $\mathrm{LVH}$}

LVH was determined by two-dimensional and M-mode echocardiography using a commercially available system (Xario 200; Toshiba Medical Systems, Tochigi, Japan). We estimated the interventricular septal thickness, LV posterior-wall thickness, relative wall thickness (the ratio of LV posterior-wall thickness to LV enddiastolic dimension), and LV mass (LVM) in linear measurements as the indices of LVH. The LVM was calculated according to the recommendations of the American Society of Echocardiology [17]. The LVM index (LVMI) was obtained by dividing the LVM by the body surface area (BSA). The BSA was calculated using the formula: $0.0001 \times 71.84 \times(\text { weight }[\mathrm{kg}])^{0.425} \times(\text { height }[\mathrm{cm}])^{0.725}$ [18]. The aortic root diameter was measured at the diastole based on an M-mode recording at the level of the sinus of Valsalva [19].

\section{Central Hemodynamics Measurements}

After the echocardiographic measurements, we performed oscillometric measurements of the central hemodynamics using the Mobil-O-Graph. Pulse-wave analysis was performed as described previously [15]. Briefly, the measurements were obtained in a quiet and temperature-controlled $\left(22-25^{\circ} \mathrm{C}\right)$ room in a seated position. The arm circumference was measured and recorded to select the correct cuff size $(20-24 \mathrm{~cm}$ or $24-32 \mathrm{~cm})$. A smaller cuff was used when the arm circumference was $24 \mathrm{~cm}$. After measuring the 
Table 1. Clinical and hemodynamic characteristics of the participants

\begin{tabular}{|c|c|c|c|c|}
\hline Parameter & $\begin{array}{l}\text { Total } \\
(n=100)\end{array}$ & $\begin{array}{l}\text { Men } \\
(n=48)\end{array}$ & $\begin{array}{l}\text { Women } \\
(n=52)\end{array}$ & $\begin{array}{l}p \\
\text { (men vs. women) }\end{array}$ \\
\hline Age, yrs & $67.5(54.0-75.0)$ & $65.0(50.3-74.8)$ & $70.0(59.5-76.0)$ & 0.169 \\
\hline $\mathrm{BMI}, \mathrm{kg} / \mathrm{m}^{2}$ & $24.1 \pm 3.4$ & $24.9 \pm 3.5$ & $23.5 \pm 3.1$ & 0.035 \\
\hline$B S A, m^{2}$ & $1.62 \pm 0.19$ & $1.77 \pm 0.17$ & $1.49 \pm 0.10$ & $<0.001$ \\
\hline ACEI/ARB use, $n(\%)$ & $37(37)$ & $19(39)$ & $18(34)$ & 0.876 \\
\hline CCB use, $n(\%)$ & $22(22)$ & $9(18)$ & $13(25)$ & 0.753 \\
\hline$\beta$-blocker use, $n$ (\%) & $5(5)$ & $2(4)$ & $3(5)$ & 0.935 \\
\hline \multicolumn{5}{|c|}{ Hemodynamic and echocardiographic parameters } \\
\hline Heart rate, beats/min & $70.7 \pm 10.9$ & $72.1 \pm 9.9$ & $69.3 \pm 11.7$ & 0.204 \\
\hline Brachial SBP, mm Hg & $146.5 \pm 14.0$ & $144.7 \pm 15.2$ & $148.1 \pm 12.7$ & 0.225 \\
\hline Brachial DBP, mm Hg & $91.5 \pm 10.3$ & $92.6 \pm 10.1$ & $90.4 \pm 10.5$ & 0.288 \\
\hline Central SBP, mm Hg & $135.0 \pm 14.0$ & $133.2 \pm 14.1$ & $136.5 \pm 13.9$ & 0.240 \\
\hline Central AP, mm Hg & $14.4 \pm 7.8$ & $11.2 \pm 6.2$ & $17.3 \pm 8.1$ & $<0.001$ \\
\hline Central Alx, \% & $32.7 \pm 13.4$ & $27.3 \pm 10.9$ & $37.6 \pm 13.7$ & $<0.001$ \\
\hline RWT & $0.498 \pm 0.051$ & $0.496 \pm 0.052$ & $0.499 \pm 0.051$ & 0.812 \\
\hline LVM, g & $182.2 \pm 30.5$ & $202.6 \pm 24.3$ & $163.3 \pm 22.6$ & $<0.001$ \\
\hline LVMI, $\mathrm{g} / \mathrm{m}^{2}$ & $112.6 \pm 16.0$ & $115.5 \pm 15.8$ & $109.8 \pm 16.0$ & 0.076 \\
\hline Aortic root diameter, $\mathrm{mm}$ & $33.1 \pm 4.0$ & $34.9 \pm 2.0$ & $31.4 \pm 3.0$ & $<0.001$ \\
\hline
\end{tabular}

Data are presented as means \pm standard deviations if normally distributed and as medians (IQRs) if not. BMI, body mass index; BSA, body surface area; ACEI/ARB, angiotensin-converting enzyme inhibitor/angiotensin II receptor blocker; CCB, calcium channel blocker; SBP, systolic blood pressure; DBP, diastolic blood pressure; AP, augmentation pressure; Alx, augmentation index; RWT, relative wall thickness; LVM, left ventricular mass; LVMI, left ventricular mass index; IQR, interquartile range.

brachial BP, the cuff was instantly inflated, and the brachial artery pressure waves were recorded at the DBP level for $10 \mathrm{~s}$. The central pressure waveform was calculated using a generalized transfer function [11], and the calibration by the systolic/diastolic brachial BP was used [20].

The Mobil-O-Graph has been reported to overestimate the AIx for negative AIx values measured with the SphygmoCor [11]. The AIx measured by the SphygmoCor showed a high correlation $(r=$ $0.822, p<0.001$ ) with the radial AIx of the radial artery measured by applanation tonometry [21]. Therefore, the AIx measured using the Mobil-O-Graph was validated against the tonometric measurements of the radial AIx (HEM-9000AI; Omron Healthcare Co., Ltd., Kyoto, Japan) in 110 normotensive healthy individuals (age range, 21-76 years; 50 men) [15]. The results showed that the AIx measured by the Mobil-O-Graph did not present negative AIx values but moderately correlated with the radial AIx measurements by the HEM-9000AI ( $r=0.602, p<0.001)$ (Fig. 1).

\section{Statistical Analysis}

The septal-wall thickness and posterior-wall thickness along with the LVMI are the recommended and validated indices to assess the degree of abnormality of LVM [17]. Hence, we analyzed the relationship between the central hemodynamics assessed using the Mobil-O-Graph and LVH in 100 patients who showed a septalor posterior-wall thickness of $\geq 11$ and $\geq 10 \mathrm{~mm}$ in men and women, respectively.

The comparison of the categorical variables between the groups including the number of antihypertensive drugs used was per- formed using the chi-square $\left(\chi^{2}\right)$ test. The Kolmogorov-Smirnov test was used to determine the normality of the variables. Continuous data are presented as means \pm standard deviations and medians (interquartile ranges) for normally and non-normally distributed data, respectively. For comparisons between men and women, $p$ values were calculated using an unpaired or Welch $t$ test for parametric data and the Mann-Whitney $U$ test for nonparametric data. $p$ values $<0.05$ were considered significant.

Pearson or Spearman correlation coefficients were calculated for the LVMI and various parameters in the univariate analysis. Multivariate linear regression analysis was performed to assess the independent determinants of the LVMI. The AIx [6] or central SBP and the dimension of the ascending aorta [10] were reported to be independently associated with the LVMI in a small population of Japanese hypertensive patients (approximately 100). The selection of the variables was based on these previous studies and additionally included age and the heart rate. The intercorrelation among various parameters for the LVMI in the univariate analysis was analyzed to assess the variance inflation factor (VIF), and the multicollinearity was assessed using the VIF. None of the VIF values was $>4.5$, suggesting that collinearity was not likely to be a concern [22].

Pearson correlation coefficients for the LVMI and various parameters were calculated in the univariate analysis in men and women. Moreover, according to a previous study which suggested that AIx measurement is useful for predicting LVH [6], LVH was defined as an LVMI $>118 \mathrm{~g} / \mathrm{m}^{2}$ in men or $>108 \mathrm{~g} / \mathrm{m}^{2}$ in women. We performed receiver operator characteristic (ROC) curve anal- 


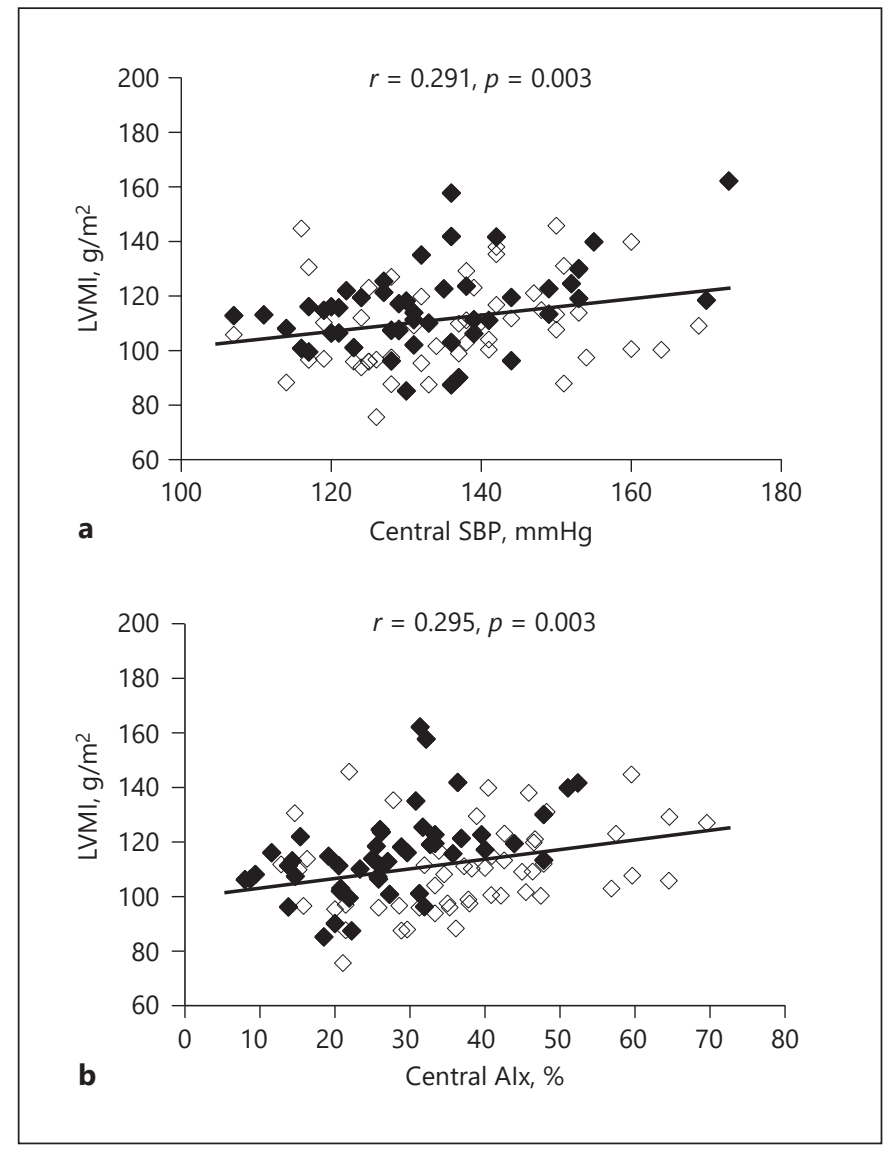

Fig. 2. Pearson correlations between the LVMI and other variables in the total cohort including both men and women among hypertensive patients. The regression line is shown as a solid line; black diamond shapes represent men and open diamond shapes represent women. SBP, systolic blood pressure; AIx, augmentation index; LVMI, left ventricular mass index.

ysis to predict the presence of LVH according to the central AIx level. The highest sum of sensitivity and specificity was identified as the cutoff value.

All analyses were performed using GraphPad Prism 6 (GraphPad Software Inc., La Jolla, CA, USA) and R version 3.2.3 (R Foundation for Statistical Computing, Vienna, Austria). The multivariate analysis was performed using $\mathrm{R}$ version 3.2.3.

\section{Results}

Table 1 shows the clinical characteristics and hemodynamic parameters of the participants. The median age of the participants was 67.5 years (interquartile range, 54.0 75.0 years). There were no significant differences between men $(n=48)$ and women $(n=52)$ with respect to the age

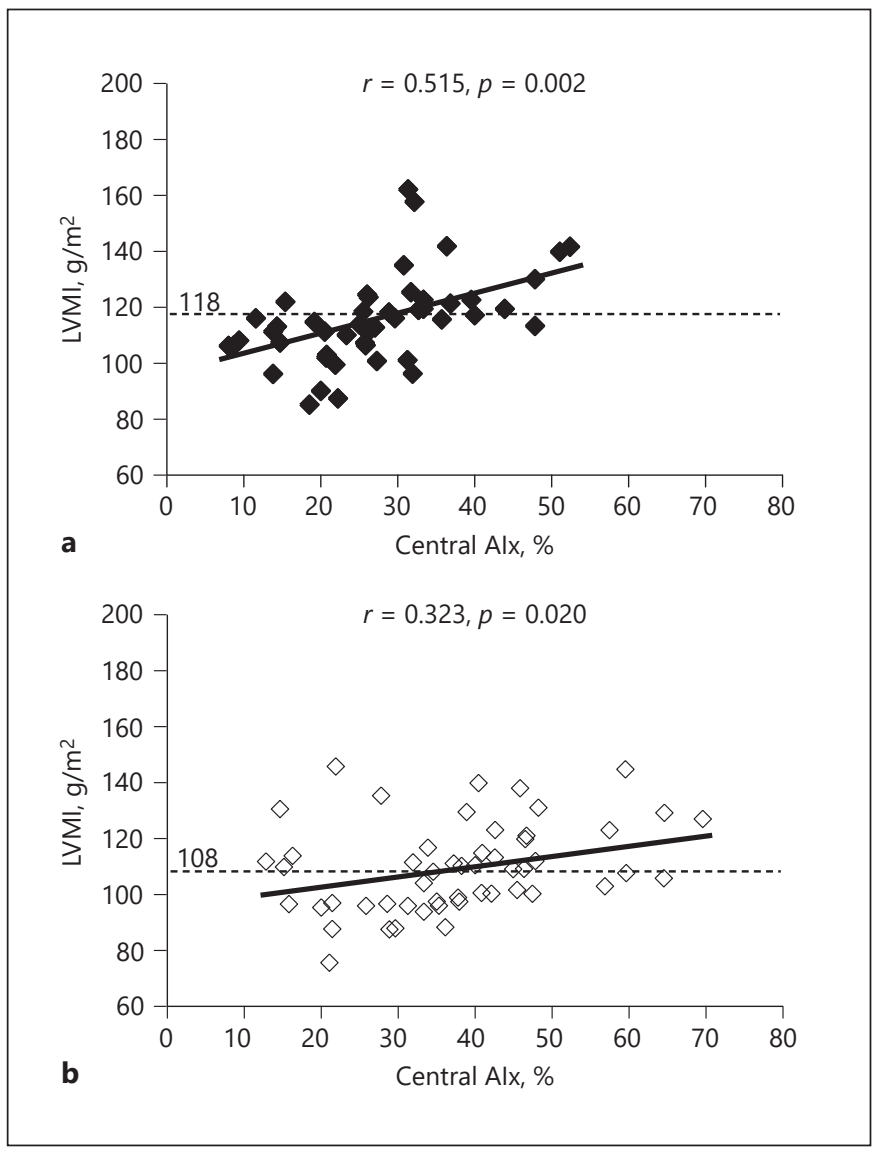

Fig. 3. Pearson correlations between the LVMI and AIx in men (a) and women (b). The regression line is shown as a solid line. The black diamond shapes represent men and open diamond shapes represent women. LVH was defined as an LVMI $>118 \mathrm{~g} / \mathrm{m}^{2}$ in men or $>108 \mathrm{~g} / \mathrm{m}^{2}$ in women. The LVH threshold is shown as a dotted line. AIx, augmentation index; LVMI, left ventricular mass index; $\mathrm{LVH}$, left ventricular hypertrophy; AIx, augmentation index.

and number of antihypertensive drugs used. Twenty-two (45.8\%) men and $24(46.2 \%)$ women were treated with at least 1 antihypertensive drug.

Body mass index and the BSA were greater in men than in women. There were no significant differences in heart rate, brachial SBP, brachial DBP, and central SBP between the sexes. The central AP and AIx were lower in men than in women, whereas the LVM and aortic root diameter were greater in men than in women. No significant difference was found between the sexes in the LVMI. The prevalence of LVH was $37.5 \%$ and $53.8 \%$ in men and women, respectively.

Spearman correlation coefficients were calculated for the LVMI and age. Pearson correlation coefficients were calculated for the LVMI and various parameters, except 
Table 2. The intercorrelations among various parameters for LVMI in univariate analysis

\begin{tabular}{|c|c|c|c|c|c|c|c|c|}
\hline Age & - & $-0.279 * *$ & $0.315^{* *}$ & $0.209 *$ & $0.533^{* * *}$ & $0.439 * * *$ & $0.270^{* *}$ & 0.100 \\
\hline Heart rate & & - & -0.053 & 0.004 & $-0.469^{* * *}$ & $-0.543^{* * *}$ & $-0.412^{* * *}$ & 0.061 \\
\hline Brachial SBP & & & - & $0.923^{\dagger, * * *}$ & $0.509 * * *$ & $0.267^{* *}$ & $0.300^{* *}$ & 0.037 \\
\hline Central SBP & & & & - & $0.500^{* * *}$ & $0.231^{*}$ & $0.291 * *$ & 0.062 \\
\hline LVMI & & & & & & & - & $0.310^{* *}$ \\
\hline Aortic root diameter & & & & & & & & - \\
\hline
\end{tabular}

Values are the Pearson or Spearman correlation coefficients ( $r$. SBP, systolic blood pressure; DBP, diastolic blood pressure; AP, augmentation pressure; Alx, augmentation index; LVMI, left ventricular mass index; VIF, variance inflation factor. ${ }^{\dagger}$ VIF value $>4.5 .{ }^{*} p<0.05$. ** $p<0.01$. ${ }^{* * *} p<0.001$ for significant correlations.

Table 3. Multivariate analysis of LVMI and other variables

\begin{tabular}{lllr}
\hline Variables & $\beta$ & Standard error for $\beta$ & $p$ value \\
\cline { 2 - 3 } & $R^{2}=0.362$; adjusted $R^{2}=0.329, p<0.001$ & \\
\hline Age, years & -0.025 & 0.124 & 0.841 \\
Heart rate, beats/min & -0.568 & 0.149 & $<0.001$ \\
Central SBP, mm Hg & 0.290 & 0.100 & 0.005 \\
Central Alx, \% & 0.120 & 0.131 & 0.361 \\
Aortic root diameter, mm & 1.355 & 0.344 & 0.001 \\
\hline
\end{tabular}

$\beta$, standardized coefficient in multivariate analysis. SBP, systolic blood pressure; Alx, augmentation index; LVMI, left ventricular mass index. age, in the univariate analysis. In the total cohort including both men and women, age ( $r=0.270, p=0.007)$, brachial SBP $(r=0.3, p=0.002)$, central SBP $(r=0.291, p=$ $0.003)$ (Fig. 2a), central AP $(r=0.310, p<0.001)$, central AIx $(r=0.295, p=0.003)$ (Fig. 2b), and aortic root diameter $(r=0.31, p=0.002)$ were positively correlated with the LVMI. The heart rate $(r=-0.412, p<0.001)$ showed a negative correlation with the LVMI in the univariate analysis.

Table 2 shows the intercorrelations among the various parameters for the LVMI in the univariate analysis. The correlation coefficients between brachial SBP and central SBP and between central AP and the AIx were $>0.889$ $(\mathrm{VIF} \approx 5)$. From the viewpoint of multicollinearity, the other side was excluded in the selection of the variables for the multivariate regression analysis. Thus, age, heart rate, central SBP, central AIx, and aortic root diameter were chosen as variables for the multivariate regression analysis. The independent determinants influencing the LVMI $\left(R^{2}=0.362\right.$; adjusted $\left.R^{2}=0.329, p<0.001\right)$ were heart rate $(\beta=-0.568 \pm 0.149, p<0.001)$, central SBP $(\beta$ $=0.290 \pm 0.100, p=0.005)$, and aortic root diameter $(\beta=$ $1.355 \pm 0.344, p=0.001)$. Age $(\beta=-0.025 \pm 0.124, p=$ $0.841)$ and central $\operatorname{AIx}(\beta=0.120 \pm 0.131, p=0.361)$ were not independently associated with the LVMI (Table 3 ).

In men, brachial $(r=0.356, p=0.013)$ and central $(r=$ $0.414, p=0.003)$ SBP significantly correlated with the LVMI in the Pearson correlation. In women, brachial SBP $(r=0.303, p=0.029)$ significantly correlated with the LVMI, but central SBP tended to correlate with the LVMI $(r=0.233, p=0.096)$. The central AIx significantly correlated with the LVMI in both men $(r=0.515, p=0.002)$ and women $(r=0.323, p=0.02)$, respectively (Fig. 3$)$. The area under the ROC curve was statistically significant in men (0.875 [95\% CI: 0.777-0.973], $p<0.001)$ but not in women (0.622 [95\% CI: 0.465-0.779], $p=0.132$ ) (Fig. 4). In men, a central AIx of $28.06 \%$ had a sensitivity of $83.3 \%$ and specificity of $80.0 \%$ for predicting the presence of $\mathrm{LVH}$. In the total cohort, the area under the ROC curve was statistically significant (0.730 [95\% CI: 0.630-0.830], 


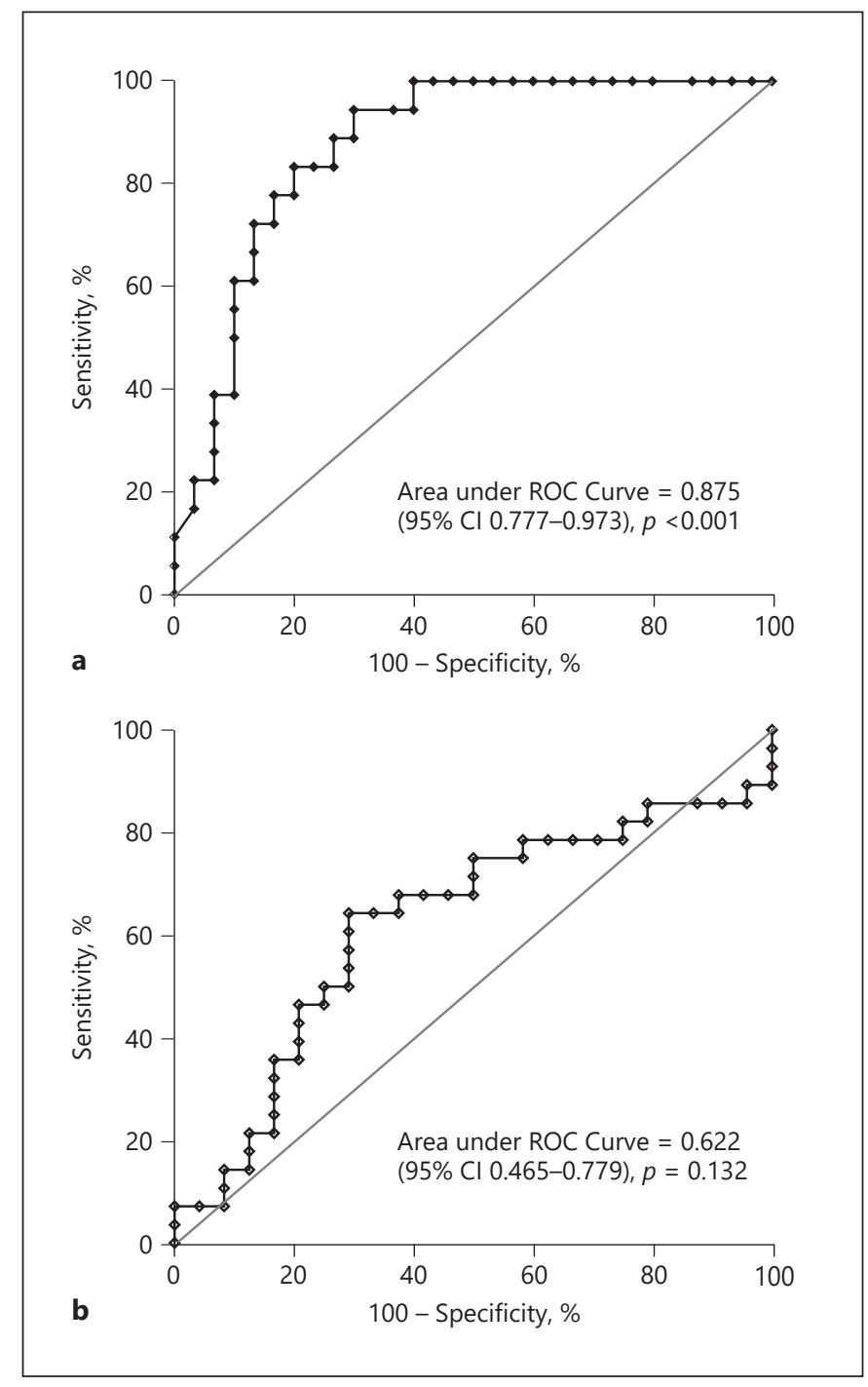

Fig. 4. ROC curves for the central AIx to predict the presence of $\mathrm{LVH}$ in men (a) and women (b). LVH, left ventricular hypertrophy; AIx, augmentation index; ROC, receiver operator characteristic.

$p<0.001$ ); however, a central AIx of $32.03 \%$ had a sensitivity of $69.6 \%$ and specificity of $68.5 \%$ for predicting the presence of LVH.

\section{Discussion}

The main findings of this study were as follows: (1) although central SBP and the AIx correlated positively with the LVMI to the same extent in the Pearson correlation analysis, there was no significant difference in the central
SBP between the sexes; however, the central AIx was found to be lower in men than in women among the hypertensive patients; (2) in multiple regression analysis of the total cohort including both men and women, central SBP was the independent determinant of the LVMI but the central AIx was not; (3) the area under the ROC curve for predictors of LVH revealed that the central AIx in men had greater predictive power $(0.875)$ than the central AIx in the total cohort (0.730). In men, a central AIx of $28.06 \%$ was the best predictor of $\mathrm{LVH}$.

With respect to AIx measurement, the Mobil-OGraph reportedly showed a low agreement with Arteriograph ( $r=0.007, p=0.924)$ and Complior $(r=0.135, p=$ $0.05)$ in a previous study [12]. In that study, the average AIx measured by the Mobil-O-Graph in 211 individuals (age, $55.1 \pm 14.1$ years; $67.8 \%$ men; $50.2 \%$ hypertensive patients) was $17.8 \pm 11.3 \%$, which seemed lower than that in our study $(32.7 \pm 13.4 \%)$ but similar to that formerly reported in another study on normotensive men (16.8 \pm 7.3\%) [15]. Another study on subjects who were almost the same age (53.9 \pm 7.9 years) as those in the aforementioned study with a normal cardiac-ankle vascular index score (no probable atherosclerosis) showed that the average AIx measured by the Mobil-O-Graph was $23.76 \pm$ $14.49 \%$ [23]. The underestimation of the AIx measurement by the Mobil-O-Graph in the former study might have influenced the low agreement with the other devices.

Central AP is calculated as the increase from the shoulder of the aortic pressure to the late systolic peak in type $A$ and $B$ waveforms or the decrease from the initial peak of the aortic pressure to the shoulder in type $\mathrm{C}$ waveforms [24]. The second positive zero crossing of the fourth derivative of the central aortic pressure defines the inflection point (corresponding to the beginning of the second wave) [25], and the Mobil-O-Graph uses the fourth derivative rule to determine the inflection point [11]. It is difficult to identify the inflection point in type $\mathrm{C}$ waveforms, which are relatively common in younger men [26]. In this study on hypertensive patients, the AP in men and women was $11.2 \pm 6.2 \mathrm{~mm} \mathrm{Hg}$ and $17.3 \pm 8.1 \mathrm{~mm} \mathrm{Hg}$, respectively. In our previous study on normotensive individuals, the $\mathrm{AP}$ in men and women was $5.5 \pm 2.8 \mathrm{~mm} \mathrm{Hg}$ and $11.0 \pm 4.7 \mathrm{~mm} \mathrm{Hg}$, respectively [15]. The AP in both sexes in this study was expected to be higher than that in normotensive men. The aortic pressure contour in this study would be type A or B waveforms; hence, it would be easier to detect the inflection point.

It was reported that the AIx in women was higher than in men among healthy individuals, and the sex-related difference in the AIx persisted even after adjusting for 
body height [21]. The mismatch between the aortic diameter and cardiac output [27] or that in distal-to-proximal arterial dimensions [28] in women is responsible for the difference in the AP between the sexes. The AIx in women was reported to remain higher than that in men among the hypertensive patients [29]. The AIx in this study was also found to be lower in men than in women.

A community sample study that was comprised of $>40 \%$ hypertensive patients showed that the AIx derived from radial applanation tonometry was associated with the LVMI in men, but not in women [30]. In this study, the area under the ROC curve of the central AIx for the presence of $\mathrm{LVH}$ was only significant in men. Thus, there appears to be a sex-specific influence of the AIx on LVH in patients with hypertension. A previous study reported that the AIx was independently correlated with the LVMI in Japanese hypertensive patients, in which $70 \%$ or more of the total cohort was male [6]. This may reflect the dominant influence of the AIx on LVH in men.

In contrast, no significant difference in the central SBP between the sexes was found in this study or in a community sample study that was comprised of $>40 \%$ hypertensive patients [30]. Central SBP was reported to be more strongly associated with $\mathrm{LVH}$ than with central pulse pressure in a large-scale study [31]. The results demonstrated that the absolute pressure, that is, systolic pressure, is more important than the relative value in stimulating LVH [31]. In Japanese patients with treated hypertension, appropriately controlled by medication, central SBP and dimension of the ascending aorta have been reported to be independent determinants of $\mathrm{LVH}$ [10]. The prevalence of $\mathrm{LVH}$ is significantly higher in patients with aortic root dilation [19]. Pearson's correlation coefficient for the LVMI and aortic root size was 0.35 in the previous study, which was similar to that in our study (0.310).

In this study, a significant relationship between the LVMI and heart rate was observed in the multivariate regression analysis. There is an inverse relationship between the heart rate and stroke volume for the same cardiac output. This basic physiological principle explains the inverse association between the LVMI and heart rate [32]. There was an inverse relationship $(r=-0.389, p<$ $0.001)$ between heart rate and stroke volume in the univariate analysis in this study (data not shown).

The present study has some limitations. First, we enrolled 22 men (45.8\%) and 24 women (46.2\%) undergoing antihypertensive treatment. A meta-analysis of 12 studies that provided data on the association between the LVMI and SBP showed significant correlations with both the central and brachial SBP [33]. In the total cohort of this study, the LVMI was significantly associated with both brachial and central SBP in the univariate analysis. The effect of the antihypertensive treatment in lowering the central and brachial SBP did not significantly affect the association between the LVMI and SBP.

Second, the patient population size was small. The study was not sufficiently powered to demonstrate the sex-specific relationships between central hemodynamics and LVH on multivariate linear regression analysis. Further examination of the sex-specific relationship between central hemodynamics assessed by the Mobil-O-Graph and $\mathrm{LVH}$ is required in a larger population.

Third, we analyzed the relationship between the central hemodynamics and LVH in hypertensive patients who showed a septal thickness or posterior wall thickness of $\geq 11$ and $\geq 10 \mathrm{~mm}$ in men and women, respectively. The average LVMI in the total cohort was $112.6 \pm 16.0 \mathrm{~g} / \mathrm{m}^{2}$, which did not seem different from that in Japanese hypertensive patients $\left(117 \pm 39 \mathrm{~g} / \mathrm{m}^{2}\right)$ as shown in another study [10].

Finally, this was a cross-sectional study. A longitudinal study will be required to describe the chronological effect of an increase in central SBP or the AIx on LVH.

In conclusion, the present study showed that central SBP, but not the central AIx, was an independent determinant of LVH in hypertensive patients in general. However, AIx measurement in men was useful for predicting the presence of LVH. These results would be in agreement with the findings of previous studies performed using radial applanation tonometry in hypertensive patients. Further examination of the sex-specific relationship between central hemodynamics assessed using the Mobil-O-Graph and hypertensive $\mathrm{LVH}$ is required in a larger population.

\section{Acknowledgments}

We thank Prof. Henk ter Keurs (Alberta, Canada) for the insightful discussions, and Ms. Satomi Kiyota, Ms. Miho Ikeda, and Ms. Sayaka Nakabayashi for their technical assistance.

\section{Statement of Ethics}

Written and opt-out informed consents were obtained from all patients included in this study. We applied the opt-out method by using a poster describing the content of the study with notifications regarding guaranteed withdrawal. The study was approved by the Ethics Committee of Sanyo-Onoda City Hospital (ref. P0002-3). 


\section{Conflict of Interest Statement}

The authors have no conflicts of interest to declare.

\section{Funding Sources}

The authors did not receive any funding.

\section{Author Contributions}

M.O. designed the study, collected data, analyzed the data, and wrote the initial draft of the manuscript. S.K. assisted in the preparation of the manuscript. M.Y. contributed to the discussion and data interpretation. T.N. and Y.H. contributed to the assistance and data interpretation.

\section{Data Availability Statement}

The data that support the findings of this study are available from the corresponding author upon reasonable request.

\section{References}

1 Bell V, Sigurdsson S, Westenberg JJ, Gotal JD, Torjesen AA, Aspelund T, et al. Relations between aortic stiffness and left ventricular structure and function in older participants in the age, gene/environment susceptibility: Reykjavik Study. Circ Cardiovasc Imaging. 2015;8(4):e003039.

2 Obayashi M, Yano M, Kohno M, Kobayashi S, Yamamoto T, Ohkusa T, et al. Effect of angiotensin II receptor antagonism on vascular hypertrophy and aortic impedance in abdominal aortic-banded rat. Am J Hypertens. 1999 Apr;12(4 Pt 1):381-7.

3 Quail MA, Short R, Pandya B, Steeden JA, Khushnood A, Taylor AM, et al. Abnormal wave reflections and left ventricular hypertrophy late after coarctation of the aorta repair. Hypertension. 2017 Mar;69(3):501-9.

4 Kobayashi S, Yano M, Kohno M, Obayashi M, Hisamatsu Y, Ryoke T, et al. Influence of aortic impedance on the development of pressure-overload left ventricular hypertrophy in rats. Circulation. 1996 Dec;94(12):3362-8

5 Zamani P, Bluemke DA, Jacobs DR, Duprez DA, Kronmal R, Lilly SM, et al. Resistive and pulsatile arterial load as predictors of left ventricular mass and geometry: the Multi-Ethnic Study of Atherosclerosis. Hypertension. 2015 Jan;65(1):85-92.

6 Hashimoto J, Watabe D, Hatanaka R, Hanasawa T, Metoki H, Asayama K, et al. Enhanced radial late systolic pressure augmentation in hypertensive patients with left ventricular hypertrophy. Am J Hypertens. 2006 Jan; 19(1):27-32.

7 Schultz MG, Davies JE, Roberts-Thomson P, Black JA, Hughes AD, Sharman JE. Exercise central (aortic) blood pressure is predominantly driven by forward traveling waves, not wave reflection. Hypertension. $2013 \mathrm{Jul} ; 62(1)$ $175-82$.

8 Schultz MG, Davies JE, Hardikar A, Pitt S, Moraldo M, Dhutia N, et al. Aortic reservoir pressure corresponds to cyclic changes in aortic volume: physiological validation in humans. Arterioscler Thromb Vasc Biol. 2014 Jul;34(7):1597-603.
9 Booysen HL, Woodiwiss AJ, Sibiya MJ, Hodson B, Raymond A, Libhaber E, et al. Indexes of aortic pressure augmentation markedly underestimate the contribution of reflected waves toward variations in aortic pressure and left ventricular mass. Hypertension. 2015 Mar;65(3):540-6.

10 Matsunaga $\mathrm{H}$, Senda S, Okuyama H, Murao $\mathrm{K}$, Inukai M, Hosomi N, et al. Comparison of central blood pressure and cardio-ankle vascular index for association with cardiac function in treated hypertensive patients. Hypertens Res. 2019;32(12):1136-42.

11 Wassertheurer S, Kropf J, Weber T, van der Giet M, Baulmann J, Ammer M, et al. A new oscillometric method for pulse wave analysis: comparison with a common tonometric method. J Hum Hypertens. 2010;24(8):498504.

12 Papaioannou TG, Thymis J, Benas D, Triantafyllidi H, Kostelli G, Pavlidis G, et al. Measurement of central augmentation index by three different methods and techniques: agreement among arteriograph, complior, and Mobil-O-Graph devices. J Clin Hypertens. 2019;21(9):1386-92.

13 Baulmann J, Schillings U, Rickert S, Uen S, Düsing R, Illyes $\mathrm{M}$, et al. A new oscillometric method for assessment of arterial stiffness: comparison with tonometric and piezo-electronic methods. J Hypertens. 2008;26(3):5238.

14 Berukstis A, Jarasunas J, Daskeviciute A, Ryliskyte L, Baranauskas A, Steponeniene R, et al. How to interpret 24-h arterial stiffness markers: comparison of 24-h ambulatory MobilO-Graph with SphygmoCor office values. Blood Press Monit. 2019;24(2):93-8.

15 Obayashi M, Kobayashi S, Kohno M, Nakashima T, Yano M. Cuff-based oscillometric measurements of central hemodynamics: factors influencing central augmentation pressure in normotensive Japanese individuals. Pulse. 2019;6(3-4):161-8.

16 Resende L, Silva MAVD, Resende EAMR, Resende JAM, Silva APMD, Casemiro ALM, et al. [Pp.01.02] correlation of pulse wave analysis parameters with left ventricular hypertrophy, intima-media-thickness and plaques in carotid arteries in a Brazilian ambulatory care. J Hypertens. 2016 Sep;34(Suppl 2):e115.

17 Marwick TH, Gillebert TC, Aurigemma G, Chirinos J, Derumeaux G, Galderisi M, et al. Recommendations on the use of echocardiography in adult hypertension: a report from the European Association of Cardiovascular Imaging (EACVI) and the American Society of Echocardiography (ASE). Eur Heart J Cardiovasc Imaging. $2015 \mathrm{Jul} ; 16(6): 577-605$.

18 Liao Y, Cooper RS, Durazo-Arvizu R, Mensah GA, Ghali JK. Prediction of mortality risk by different methods of indexation for left ventricular mass. J Am Coll Cardiol. 1997 Mar; 29(3):641-7.

19 Cuspidi C, Meani S, Fusi V, Valerio C, Sala C, Zanchetti A. Prevalence and correlates of aortic dilatation in patients with essential hypertension: relationship with cardiac and extracardiac target organ damage. J Hypertens. 2006;24(3):573-80.

20 Gotzmann M, Hogeweg M, Bauer F, Seibert FS, Rohn BJ, Mügge A, et al. The impact of calibration approaches on the accuracy of oscillometric central aortic blood pressure measurement. J Hypertens. 2020 Nov;38(11): 2154-60.

21 Kohara K, Tabara Y, Oshiumi A, Miyawaki Y, Kobayashi T, Miki T. Radial augmentation index: a useful and easily obtainable parameter for vascular aging. Am J Hypertens. 2005 Jan; 18(1 Pt 2):11S-4S.

22 Chen Q, Turban S, Miller ER, Appel LJ. The effects of dietary patterns on plasma renin activity: results from the dietary approaches to stop hypertension trial. J Hum Hypertens. 2012;26(11):664-9.

23 Gomez-Sanchez L, Garcia-Ortiz L, PatinoAlonso MC, Recio-Rodriguez JI, Frontera G, Ramos R, et al. The association between the cardio-ankle vascular index and other parameters of vascular structure and function in Caucasian adults: MARK Study. J Atheroscler Thromb. 2015;22(9):901-11. 
24 Murgo JP, Westerhof N, Giolma JP, Altobelli SA. Aortic input impedance in normal man: relationship to pressure waveforms. Circulation. 1980 Jul;62(1):105-16.

25 Kelly R, Hayward C, Avolio A, O'Rourke M. Noninvasive determination of age-related changes in the human arterial pulse. Circulation. 1989 Dec;80(6):1652-9.

26 Hughes AD, Park C, Davies J, Francis D, McG Thom SA, Mayet J, et al. Limitations of augmentation index in the assessment of wave reflection in normotensive healthy individuals. PLoS One. 2013;8(3):e59371.

27 Mitchell GF, Gudnason V, Launer LJ, Aspelund T, Harris TB. Hemodynamics of increased pulse pressure in older women in the community-based Age, Gene/Environment Susceptibility-Reykjavik Study. Hypertension. 2008 Apr;51(4):1123-8.
28 Cecelja M, Jiang B, McNeill K, Kato B, Ritter J, Spector T, et al. Increased wave reflection rather than central arterial stiffness is the main determinant of raised pulse pressure in women and relates to mismatch in arterial dimensions: a Twin Study. J Am Coll Cardiol. 2009 Aug;54(8):695-703.

29 Cameron JD, McGrath BP, Dart AM. Use of radial artery applanation tonometry and a generalized transfer function to determine aortic pressure augmentation in subjects with treated hypertension. J Am Coll Cardiol. 1998 Nov;32(5):1214-20.

30 Sibiya MJ, Norton GR, Hodson B, Redelinghuys M, Maseko MJ, Majane OH, et al. Gender-specific contribution of aortic augmentation index to variations in left ventricular mass index in a community sample of African ancestry. Hypertens Res. 2014;37(11):1021-7.
31 Roman MJ, Okin PM, Kizer JR, Lee ET, Howard BV, Devereux RB. Relations of central and brachial blood pressure to left ventricular hypertrophy and geometry: the Strong Heart Study. J Hypertens. 2010;28(2):384-8.

32 Saba MM, Ibrahim MM, Rizk HH. Gender and the relationship between resting heart rate and left ventricular geometry. J Hypertens. 2001;19(3):367-73.

33 Kollias A, Lagou S, Zeniodi ME, Boubouchairopoulou N, Stergiou GS. Association of central versus brachial blood pressure with target-organ damage. Systematic review and meta-analysis. Hypertension. 2016 Jan;67(1): $183-90$ 